\title{
Stacking-Order Effect on Spin-Orbit Torque, Spin Hall Magnetoresistance, and Magnetic Anisotropy in $\mathrm{Ni}_{81} \mathrm{Fe}_{19}-\mathrm{IrO} \mathrm{O}_{2}$ Bilayers
}

\author{
Kohei Ueda $\odot,{ }^{1,2, *}$ Naoki Moriuchi, ${ }^{1}$ Kenta Fukushima, ${ }^{1}$ Takanori Kida $\odot,{ }^{3}$ Masayuki Hagiwara,${ }^{3}$ and \\ Jobu Matsuno (1) 1,2 \\ ${ }^{1}$ Department of Physics, Graduate School of Science, Osaka University, Osaka 560-0043, Japan \\ ${ }^{2}$ Center for Spintronics Research Network, Graduate School of Engineering Science, Osaka University, \\ Osaka 560-8531, Japan \\ ${ }^{3}$ Center for Advanced High Magnetic Field Science, Graduate School of Science, Osaka University, \\ Osaka 560-0043, Japan
}

(Received 24 April 2021; revised 1 August 2021; accepted 8 September 2021; published 22 September 2021)

\begin{abstract}
The $5 d$ transition-metal oxides are an intriguing platform to demonstrate efficient charge-to-spin-current conversion due to a unique electronic structure dominated by strong spin-orbit coupling. Here, we report on the stacking-order effect of spin-orbit torque (SOT), spin-Hall magnetoresistance, and magnetic anisotropy in bilayer $\mathrm{Ni}_{81} \mathrm{Fe}_{19}-5 d$ iridium oxide, $\mathrm{IrO}_{2}$. While all $\mathrm{IrO}_{2}$ and $\mathrm{Pt}$ control samples exhibit large dampinglike SOT generation, stemming from the efficient charge-to-spin-current conversion, the magnitude of the SOT is larger in the $\mathrm{IrO}_{2}(\mathrm{Pt})$ bottom sample than in the $\mathrm{IrO}_{2}(\mathrm{Pt})$ top one. The fieldlike SOT has an even more significant stacking-order effect, resulting in an opposite sign in the $\mathrm{IrO}_{2}$ samples in contrast to the same sign in the Pt samples. Furthermore, we observe that the magnetic anisotropy energy density and the anomalous Hall effect are increased in the $\mathrm{IrO}_{2}(\mathrm{Pt})$ bottom sample, suggesting enhanced interfacial perpendicular magnetic anisotropy. Our findings highlight the significant influence of the stacking order on spin transport and the magnetotransport properties of Ir oxide-ferromagnet systems, providing useful information for the design of SOT devices, including $5 d$ transition-metal oxides.
\end{abstract}

DOI: 10.1103/PhysRevApplied.16.034039

\section{INTRODUCTION}

Interfaces composed of a ferromagnet (FM) and a nonmagnet (NM) with strong spin-orbit coupling (SOC) have been an important arena to explore spin-current-related phenomena [1]. One of the interesting subjects is spin-orbit torque (SOT), which is generated by an in-plane current through the NM/FM bilayers and subsequent spin accumulation through charge-to-spin-current conversion [2]. The relevant conversion mechanism can be the spin Hall effect (SHE) of the bulk NM [3] and/or the interface RashbaEdelstein effect (REE) [4], while both effects are triggered by the strong SOC. Irrespective of these origins, the SOT is highly sensitive to interface properties, since spin accumulation occurs at the interface; the SOT is established as a powerful way to clarify the interface properties [2,5-22]. The NM/FM interfaces can be examined by other techniques, such as the spin Hall magnetoresistance (SMR) [23-25], spin pumping [26,27], spin-torque ferromagnetic resonance [6], perpendicular magnetic anisotropy (PMA) [28-30], the anomalous Hall effect (AHE) [31-36], and the magnetic proximity effect [37-39]. The validity of

\footnotetext{
*kueda@phys.sci.osaka-u.ac.jp
}

these measurements is widely demonstrated by using $5 d$ transition metals as the NM, e.g., Pt, Ta, and W [5-39].

One class of spintronic materials, the $5 d$ transitionmetal oxides, is of special interest because of its unique electronic structure; the Fermi surface is dominated only by $5 d$ electrons with strong SOC, in contrast to that of $5 d$ transition metals dominated by both $5 d$ and $6 s$ electrons. Recently, spintronic studies related to conductive Ir oxides have been attracting much attention [40-48]; several research groups have experimentally proven sizable SOT via the SHE in epitaxial $\mathrm{SrIrO}_{3}$ [43-45], epitaxial $\mathrm{IrO}_{2}$ [48], and amorphous $\mathrm{IrO}_{2}$ [47]. These reports highlight efficient charge-to-spin-current conversion of the Ir oxides, suggesting that the unique electronic structure inherent to the $5 d$ orbital plays a crucial role in the spintransport mechanism. While Ir oxides thus are a potential component for spintronic devices, there remains unsolved issues related to device design when we combine Ir oxides with the FM. One of the most important issues is the stacking order of the bilayer structure, which greatly influences the interface properties. In typical Pt/FM/Pt layers with two interfaces, for instance, one of the interfaces is indeed found to largely contribute to PMA $[30,49,50]$ and SOT [19]. Thus, inverting the stacking order in the bilayer is an 
effective approach, allowing us to further understand and control interfacial-driven properties, such as SOT, SMR, the AHE, and PMA.

Here, we report that the stacking order of bilayers of $\mathrm{Ni}_{81} \mathrm{Fe}_{19}(\mathrm{Py}) / \mathrm{IrO}_{2}$ strongly influences spin transport and electrical and magnetic properties evaluated by SOT, SMR, and magnetotransport measurements. We choose amorphous $\mathrm{IrO}_{2}$, since it can be easily incorporated into devices due to its simplicity of deposition. The stacking order affects the dampinglike (DL) SOT and the PMA. Their magnitudes are larger in the $\mathrm{IrO}_{2}$-bottom sample than in the $\mathrm{IrO}_{2}$-top one, whereas the same behavior is observed in Pt control samples. The fieldlike (FL) SOT shows an even more drastic stacking-order effect, resulting in an opposite sign between the $\mathrm{IrO}_{2}$-bottom and $\mathrm{IrO}_{2}$-top samples, in contrast to the same sign in the Pt samples. These findings highlight the significance of the stacking order in designing spintronic devices, including $5 d$ transition-metal oxides.

\section{EXPERIMENT}

We grow two types of samples, comprising of substrate $/ \mathrm{TaO}_{x}(1.5) / \mathrm{Ni}_{81} \mathrm{Fe}_{19}(3) / \mathrm{IrO}_{2}(10)$ and substrate/ $\mathrm{IrO}_{2}(10) / \mathrm{Ni}_{81} \mathrm{Fe}_{19}(3) / \mathrm{TaO}_{x}(2.3)$ on thermally oxidized $\mathrm{Si}$ substrates by magnetron sputtering [Fig. 1(a)]. These samples are labeled as $\mathrm{IrO}_{2}-T$ and $\mathrm{IrO}_{2}-B$. The numbers in parentheses indicate the layer thicknesses in nanometers. The $\mathrm{Ni}_{81} \mathrm{Fe}_{19}$ alloy is a soft ferromagnet with in-plane magnetic anisotropy, referred to as permalloy (Py). Before deposition of the Py layer, we eliminate surface oxidation of the Py target with sufficient sputtering time. The $\mathrm{TaO}_{x}$ layers are obtained by deposition of $\mathrm{Ta}$ and subsequent oxidation from the $\mathrm{SiO}_{2}$ substrate $\left(\mathrm{IrO}_{2}-T\right)$ and air $\left(\mathrm{IrO}_{2}-B\right)$; in this experimental design, we expect to prevent the oxidation of Py surfaces at the side opposite to $\mathrm{IrO}_{2}$. Considering the previous study [18] and our sample structures, the $\mathrm{TaO}_{x}$ layers would not influence interfacial roughness of the bilayer, suggesting that the layers are not crucial in spin transport and magnetotransport effects. We expect that current shunting by the $\mathrm{TaO}_{x}$ layers can be neglected due to their high resistance. Amorphous $\mathrm{IrO}_{2}$ is grown by a reactive sputtering method at a rate of $\mathrm{Ar}: \mathrm{O}_{2}=8: 2$ [47]. The surface of the Py layer is exposed to $\mathrm{Ar}-\mathrm{O}_{2}$ gas only in the $\mathrm{IrO}_{2}-T$ sample, giving rise to additional oxidation of the Py layer, as discussed in Sec. III A. We also prepare control samples including $\mathrm{Pt}$, namely, substrate $/ \mathrm{TaO}_{x}(1.5) / \mathrm{Ni}_{81} \mathrm{Fe}_{19}$ (3)/ Pt(6) (Pt-T) and substrate $/ \mathrm{Pt}(6) / \mathrm{Ni}_{81} \mathrm{Fe}_{19}(3) / \mathrm{TaO}_{x}(2.3)(\mathrm{Pt}-B)$. All of the metal and oxide layers are grown at a total deposition pressure of about $0.4 \mathrm{~Pa}$, while no annealing treatment before or after deposition is provided.

The Hall bar structure, fabricated using photolithography and postdeposition lift-off, has channel dimensions of $10 \mu \mathrm{m}$ in width $(w)$ and $30 \mu \mathrm{m}$ in length $(l)$, as illustrated in Fig. 1(a). I indicates ac or de current flows producing the current-driven SOT, which has two components with different symmetries, namely, DL- and FL SOTs, corresponding to effective fields $B_{\mathrm{DL}}$ and $B_{\mathrm{FL}}$, respectively. These fields represent $B_{\mathrm{DL}} \| \sigma \times M$ and $B_{\mathrm{FL}} \| \sigma$, where the direction of accumulated spin, $\sigma$, at the interface is along $y$. The Hall bars are mounted on a motorized stage, allowing for in-plane $(\phi)$ and out-of-plane $(\theta)$ rotation, with the angle and coordinate system shown in Fig. 1(a). The samples Are placed in an electromagnet producing fields in the range of 0.05 to $1.5 \mathrm{~T}$. The experiments are performed at room temperature using an ac current, $I_{\mathrm{ac}}=$ $\sqrt{2} I_{\text {rms }} \sin (2 \pi f t)$, with $f=13 \mathrm{~Hz}$ for resistivity, anomalous Hall, and harmonic Hall measurements and using a dc current, $I_{\mathrm{dc}}$, for magnetoresistance measurements. We set $I_{\mathrm{rms}}=50 \mu \mathrm{A}$ for the anomalous Hall measurements, $I_{\mathrm{rms}}=1-2 \mathrm{~mA}$ for harmonic Hall measurements, and $I_{\mathrm{dc}}=500 \mu \mathrm{A}$ for magnetoresistance measurements.

\section{RESULTS}

\section{A. Electrical transport and magnetic properties}

We examine the saturation magnetization $\left(M_{s}\right)$ of each bilayer by measuring the in-plane hysteresis loop using a superconducting quantum interference device magnetometer. Figure 1(b) shows $M_{s}$ for all samples. We find that the $M_{s}$ values of the Pt samples are larger than those of the $\mathrm{IrO}_{2}$ samples; we estimate $M_{S}(\mathrm{Pt})$ of $5.80-6.05 \times 10^{5} \mathrm{~A} / \mathrm{m}$ and $M_{S}\left(\mathrm{IrO}_{2}\right)$ of $4.90-5.35 \times 10^{5} \mathrm{~A} / \mathrm{m}$. We associate the reduction in the $\mathrm{IrO}_{2}$ samples with partial oxidation of the Py layer. In particular, the reduction of $M_{s}$ is larger in the $\mathrm{IrO}_{2}-T$ sample than in the $\mathrm{IrO}_{2}-B$ sample. Compared with the $\mathrm{Pt}$ samples, the reduction is roughly $20 \%$ in the $\mathrm{IrO}_{2}-T$ sample. This corresponds to the oxidized and probably nonmagnetic Py thickness of $0.6 \mathrm{~nm}$, while the thickness is estimated to be $0.2 \mathrm{~nm}$ for the $\mathrm{IrO}_{2}-B$ sample. Figure 1(c) displays the sheet resistance $\left(R_{\mathrm{sq}}\right)$ for all samples. We observe a higher $R_{\mathrm{sq}}$ in the $\mathrm{IrO}_{2}$ samples than that in the Pt samples, resulting from more resistive $\mathrm{IrO}_{2}$ than Pt. By focusing on the stacking order, the $R_{\mathrm{sq}}$ value in the $\mathrm{IrO}_{2}-T$ sample is $14 \%$ larger than that in the $\mathrm{IrO}_{2}-B$ one, while $R_{\mathrm{sq}}$ is almost the same for the Pt- $T$ and Pt- $B$ samples. This difference comes from the oxidation effect of the $\mathrm{IrO}_{2}-T$ sample introduced by the abovementioned difference in deposition conditions, depending on the stacking order; oxidation of the Py surface in the $\mathrm{IrO}_{2}-T$ sample is greater than that in the $\mathrm{IrO}_{2}-B$ one. To discuss the oxidation effect, we calculate the resistivity $(\rho)$ within a parallel resistor model. According to $\rho\left(\mathrm{IrO}_{2}\right) \sim 370 \mu \Omega \mathrm{cm}$ and $\rho$ $(\mathrm{Pt}) \sim 26 \mu \Omega \mathrm{cm}$ from our previous work [47] and the measured $R_{\text {sq }}$, we extract $\rho$ (Py) $\sim 105 \mu \Omega \mathrm{cm}$ for the $\mathrm{IrO}_{2}-T$ sample, $\rho$ (Py) $\sim 87 \mu \Omega \mathrm{cm}$ for the $\mathrm{IrO}_{2}-B$ one, and $\rho$ (Py) $\sim 83 \mu \Omega \mathrm{cm}$ for the Pt samples. The resistive Py in the $\mathrm{IrO}_{2}$ samples results from the reduced Py thickness due 
(a)

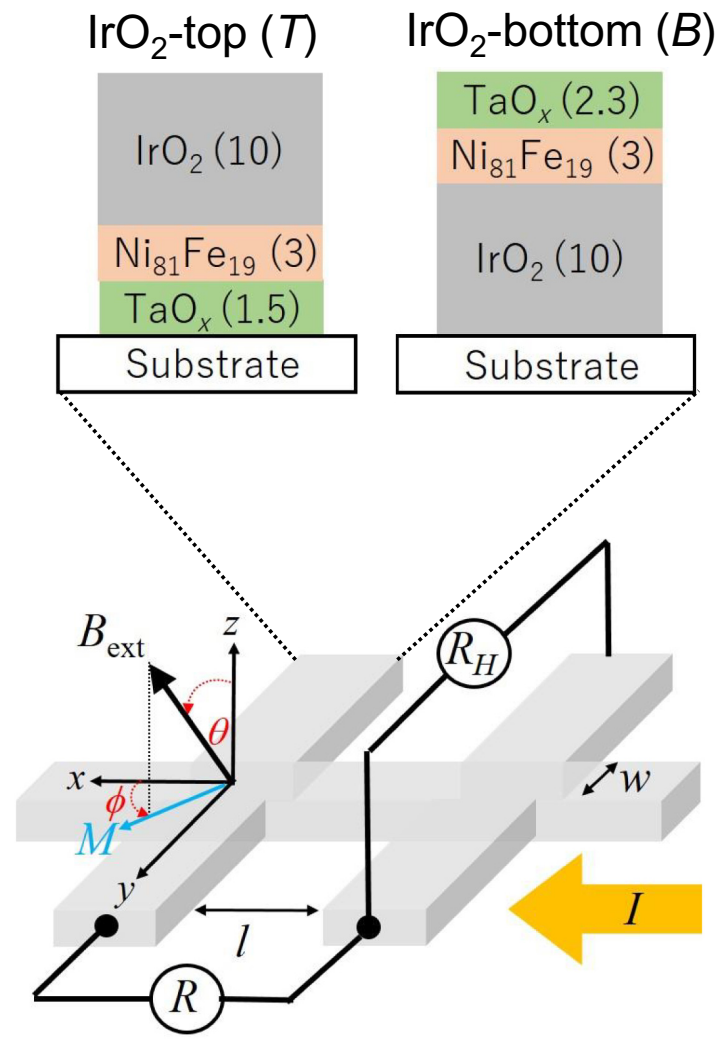

(b)

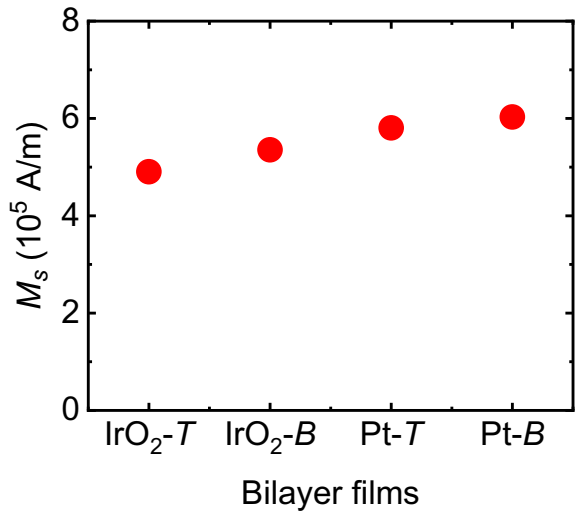

(c)

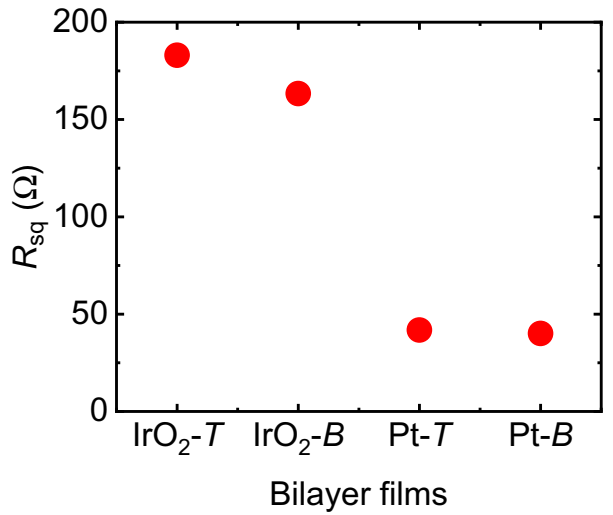

FIG. 1. (a) Top, cross section of bilayer films labeled as $\operatorname{IrO}_{2}$ top $(T), \mathrm{Py}(3) / \mathrm{IrO}_{2}(10)$ and $\operatorname{IrO}_{2}$ bottom $(B), \operatorname{IrO} 2(10) / \mathrm{Py}(3)$. Bottom, patterned device structure with two Hall bars, electrical detection, and coordinate system. (b) Saturation magnetization and (c) sheet resistance for samples with $\mathrm{IrO}_{2}-T$ and $\mathrm{IrO}_{2}-B$, including $\mathrm{Pt}$ control samples of $\mathrm{Py}(3) / \mathrm{Pt}(6)$ and $\mathrm{Pt}(6) / \mathrm{Py}(3)$.

to oxidation. Assuming that oxidized Py is fully insulating, the thickness of the oxidized Py layer is calculated to be about 0.6 and $0.2 \mathrm{~nm}$ for the $\mathrm{IrO}_{2}-T$ and $\mathrm{IrO}_{2}-B$ samples, respectively. These values are consistent with those obtained from $M_{s}$, and therefore, we consider that Py in the $\mathrm{IrO}_{2}-T$ sample is significantly more oxidized than Py in the $\mathrm{IrO}_{2}-B$ one.

We measure the anomalous Hall resistance $\left(R_{\mathrm{AHE}}\right)$ and effective anisotropy field $\left(B_{k}^{\text {eff }}\right)$ by sweeping a perpendicular magnetic field $\left(B_{z}\right)$ at $\theta=0^{\circ}$ to determine the perpendicular magnetic anisotropy energy density $(K)$ by using the following relation: $K=\left(M_{s} / 2\right)\left(\mu_{0} M_{s}-B_{k}^{\text {eff }}\right)$ [51]. Figure 2(a) shows the representative results for the $\mathrm{IrO}_{2}-T$ sample. Since the first harmonic Hall resistance $\left(R_{H}^{1 \omega}\right)$ is saturated in the high-field regime, we obtain $R_{\mathrm{AHE}}$ by subtraction of two extrapolated high-field slopes (black lines). $B_{k}^{\text {eff }}$ is estimated by subtraction of two intersections (black dotted line) given by a linear fitting of the lowfield data with a black line. $K$ for all samples is displayed in Fig. 2(b). $K$ of the $\operatorname{IrO}_{2}(\mathrm{Pt})-B$ sample is found to be significantly increased by a factor of about 6 compared with the corresponding $\mathrm{IrO}_{2}(\mathrm{Pt})-T$ sample. Since the bottom Pt layer is known to play a dominant role in strong PMA in the typical Pt/FM bilayers [10,12,16,18,49,52], we relate the substantial difference to enhanced interfacedriven PMA in the Pt- $B$ sample, resulting in large $K$. The correlation between PMA and the contribution of the orbital moment has been experimentally found [49,52]; strong PMA with a large orbital moment is induced when FM on Pt is prepared, contrary to FM underneath Pt [49]. We then focus on Fig. 2(c), which displays $R_{\mathrm{AHE}}$ for all samples. Similar to the trend of $K, R_{\mathrm{AHE}}$ of the $\mathrm{IrO}_{2}(\mathrm{Pt})-B$ sample is increased by about $40 \%$ compared with that of the $\mathrm{IrO}_{2}(\mathrm{Pt})-T$ one. We point out that the AHE is known to consist of bulk and interface contributions [31-39,53-56]. The former is the resistivity effect [36], while the latter includes several origins, such as the magnetic proximity effect [37-39], intermixing [53], and the interface SOC [31,54-56]; the SOC also plays a crucial role in strong PMA [31,54-56]. The observed enhancement of AHE in the $\mathrm{IrO}_{2}(\mathrm{Pt})-B$ sample cannot be explained by the resistivity effect [36], since the $\mathrm{IrO}_{2}-B$ sample has a lower $R_{\mathrm{sq}}$ 
(a)

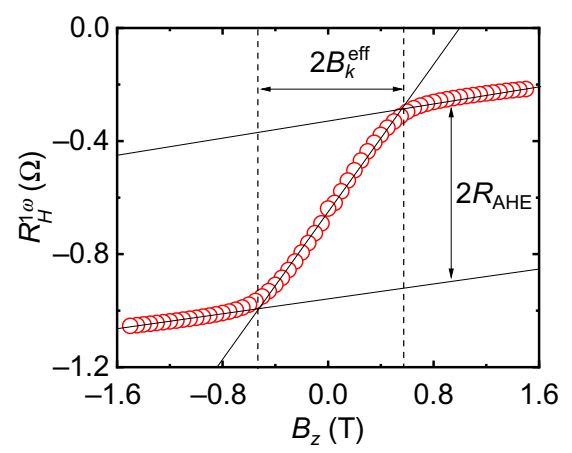

(b)

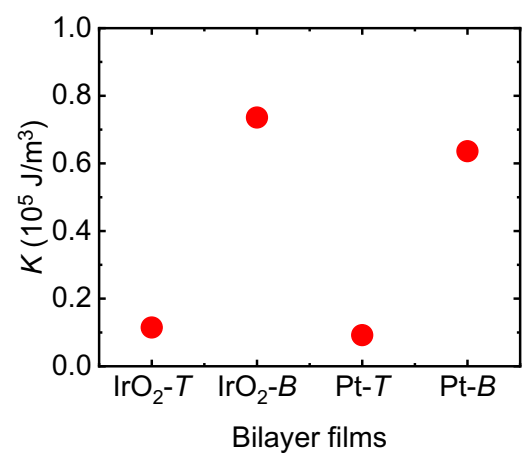

(c)

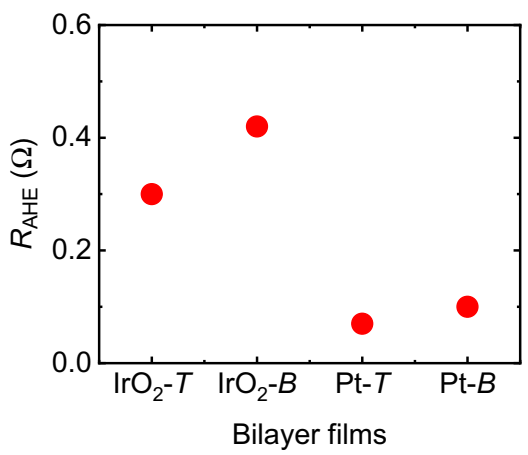

FIG. 2. (a) Hall resistance of $\mathrm{IrO}_{2}-T$ sample as a function of perpendicular magnetic field at $\theta=0^{\circ}$. (b) Perpendicular magnetic anisotropy energy density and (c) anomalous Hall resistance for all samples extracted from measurements, similar to that shown in (a).

value than that in the $\mathrm{IrO}_{2}-T$ one [see Fig. 1(c)]. Considering that the correlation of $K$ and $R_{\mathrm{AHE}}$ for the $\mathrm{IrO}_{2}(\mathrm{Pt})-B$ sample is obvious in Figs. 2(b) and 2(c), our data indicate a large interface contribution to the $\mathrm{IrO}_{2}(\mathrm{Pt})-B$ sample. Whereas such an interface-driven AHE and PMA in the amorphous $\mathrm{IrO}_{2} / \mathrm{FM}$ system remains unclear at this stage, the contribution of the orbital moment is likely to be similar to that of $\mathrm{Pt}$, considering the observed similarity between the $\mathrm{IrO}_{2}$ and Pt samples.

\section{B. Magnetoresistance measurements}

We present a description of the magnetoresistance (MR) measurements. The first-harmonic longitudinal resistance $\left(R_{L}^{1 \omega}\right)$, which is equivalent to standard dc measurements, can be typically expressed by the following general form [14]:

$$
R_{L}^{1 \omega}=R_{0}-\Delta R_{z x} \sin ^{2} \theta \cos ^{2} \phi+\Delta R_{z y} \sin ^{2} \theta \sin ^{2} \phi,
$$

where $R_{0} \equiv R(M \| x), \Delta R_{z x}$ is the resistance difference when $M$ sufficiently saturates along the $z$-axis and $x$-axis directions with $B_{\text {ext }}$, and $\Delta R_{z y}$ is the resistance difference when $M$ sufficiently saturates along the $z$-axis and $y$-axis directions with $B_{\text {ext }}$. We also define $\Delta R_{x y}$, which is the resistance difference between $M$ at $x$ and $y$ directions, corresponding to $\Delta R_{z y}-\Delta R_{z x}$.

We measure $R_{L}^{1 \omega}$ by rotating a sample with a fixed $B_{\text {ext }}=1.35 \mathrm{~T}$ in three orthogonal planes, as shown in Fig. 3(a). The applied $B_{\text {ext }}$ is large enough to saturate $M$ along all coordinate axes, allowing us to characterize magnetoresistances, such as $\Delta R_{z y}, \Delta R_{x y}$, and $\Delta R_{z x}$. Examination of these planes is essential to distinguish the contribution of SMR and anisotropic magnetoresistance (AMR). The $z-y$ scan illustrates the SMR, which is magnetoresistance due to asymmetry between absorption and reflection of the spin current generated from the bulk SHE in the NM layer [23-25]. Accordingly, this contribution gives higher resistance at $M \| z$ and lower resistance at $M \| y$, resulting in $\Delta R_{z y} \approx \Delta R_{x y}>0$ and $\Delta R_{z x} \approx 0$. The $z-x$ scan illustrates the AMR, originating from the enhanced scattering of conduction electrons from the localized $d$ orbitals ( $s$ $d$ scattering) in the bulk FM [57]. This contribution gives a higher resistance at $M \| x$ and lower resistance at $M \| z$, resulting in $\Delta R_{z x} \approx \Delta R_{x y}>0$ and $\Delta R_{z y} \approx 0$. The $x-y$ scan includes both SMR and AMR contributions in the NM contacted with the conducting ferromagnet. Figure 3(b) shows the representative magnetoresistance of the $\mathrm{IrO}_{2}-T$ sample for three planes. To determine the MR ratio, the amplitudes $\left(\Delta R_{z y}, \Delta R_{z x}\right.$, and $\left.\Delta R_{x y}\right)$ are independently obtained using Eq. (1) to fit the respective MR curves. We confirm that the relationship $\Delta R_{x y}=\Delta R_{z y}-\Delta R_{z x}$ is within the error bars, indicating that our fitting is valid to determine each amplitude of the MR ratio. Notably, a slight discrepancy between data and fitting in the $z-y$ and $z-x$ planes is observed, possibly from a contribution of higher-order MR stemming from the texture effect [58].

Figures 3(c)-3(e) summarize normalized magnetoresistances for all samples, defined as $\Delta R_{z y, z x, x y} / R_{0}$. The largest magnetoresistance appears in the $x-y$ planes for all samples, resulting from the combination of SMR and AMR. Here, we point out recent MR studies on bilayers that have been a subject of ongoing debate [58-63]. The possible contributions are the anisotropic interface MR [58,59], the anomalous Hall MR [62], the absorption of spin current in the FM layer $[25,61]$, and spin-current generation from the FM layer $[60,61,63]$. Considering the above effects, the use of a conducting FM makes the SMR effect more complicated than the SMR observed in Pt contacted with magnetic insulators [23]. The magnitude of $\Delta R_{z y} / R_{0}$ is larger than that of $\Delta R_{z x} / R_{0}$, indicating that the SMR contribution is dominant in all samples; we expect that $\Delta R_{z y} / R_{0}$ mostly includes contributions of the SMR, as discussed in Sec. III D. The larger SMR for the Pt sample than the $\mathrm{IrO}_{2}$ samples is observed as well. This is ascribed to a current-shunting effect because of more current flow in Pt than that in $\mathrm{IrO}_{2}$. We also find that both SMR and 
(a)

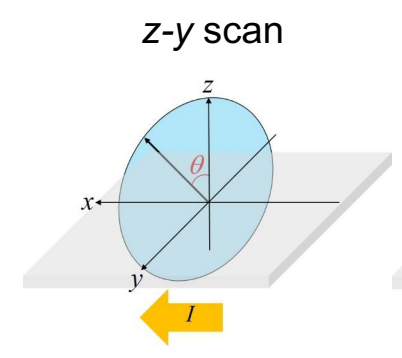

(c)

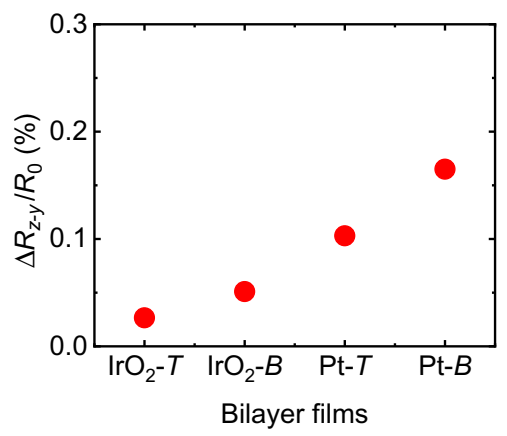

$z-x$ scan

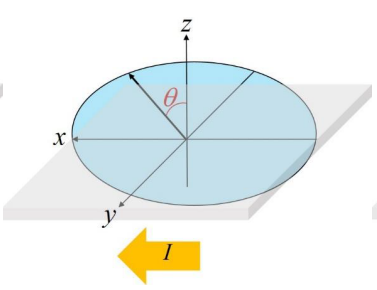

(d) (b)

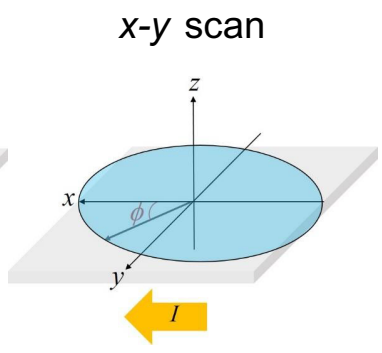

(e)
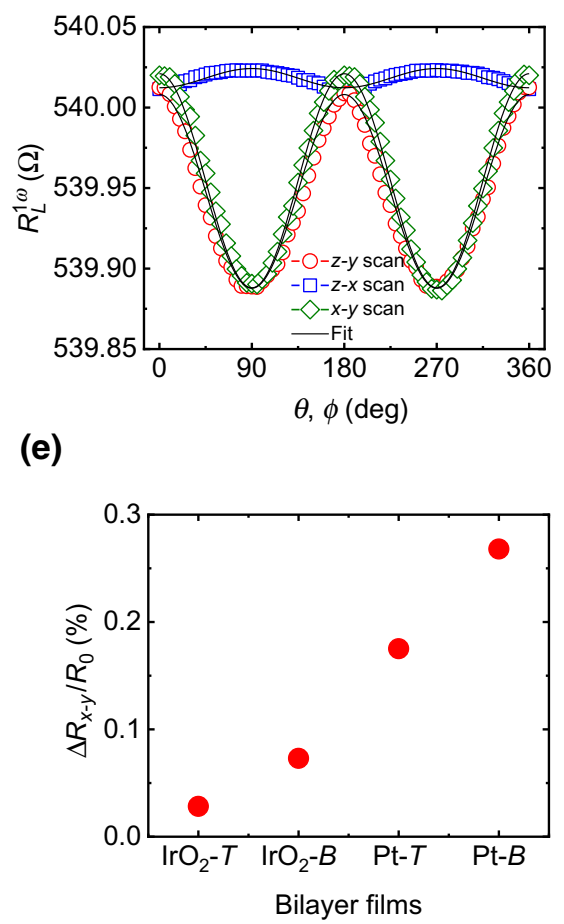

FIG. 3. (a) Illustration of rotation planes. (b) First-harmonic longitudinal resistance of $\mathrm{IrO}_{2}-T$ sample measured by rotating samples with a fixed external field of $1.3 \mathrm{~T}$. Black line is the result of fitting using Eq. (1). (c)-(e) Magnetoresistance expressed as percentage in three planes for all samples.

AMR are enhanced in the $\mathrm{IrO}_{2}(\mathrm{Pt})$-bottom samples. If $\Delta R_{z x} / R_{0}$ is fully contributed to by AMR of the bulk FM, the stacking-order effect should be opposite, according to the current-shunting effect. While this strongly suggests that the observed $\Delta R_{z x} / R_{0}$ includes some interface contribution, we do not discuss $\Delta R_{z x}$ in detail, since it is difficult to pin down the role of the interface at the current stage. However, given a report on the MR in bilayer systems including $\mathrm{IrO}_{2}$, it serves as a good starting point to clarify complicated MR with further study. Unlike $\Delta R_{z x}, \Delta R_{z y}$ is well related to the DL SOT efficiency; by analyzing the efficiency through the conventional SMR model, we elucidate the stacking-order effect in Sec. III D.

\section{Harmonic Hall measurements}

We quantify DL SOT and FL SOT by performing harmonic Hall measurements as described in Refs. [9$11,14]$. While $R_{H}^{1 \omega}$ is equivalent to the conventional Hall resistance, the second-harmonic resistance, $R_{H}^{2 \omega}$, provides information about the SOT; an injection of $I_{\mathrm{ac}}$ produces SOTs that cause a small modulation of magnetization about its equilibrium position against the magnetic field. In the analysis of the harmonic Hall measurement established by Avci et al. [11], the following relationship is used when magnetization sufficiently lies in plane $\left(\theta=90^{\circ}\right)$ :

$$
\begin{aligned}
R_{H}^{1 \omega}= & R_{\mathrm{PHE}} \sin 2 \phi \sin ^{2} \theta, \\
R_{H}^{2 \omega}= & -\left(R_{\mathrm{AHE}} \frac{B_{\mathrm{DL}}}{B_{k}^{\mathrm{eff}}+B_{\mathrm{ext}}}+R_{\nabla T}\right) \cos \phi \\
& +2 R_{\mathrm{PHE}} \frac{B_{\mathrm{FL}}+B_{\mathrm{Oe}}}{B_{\mathrm{ext}}}\left(2 \cos ^{3} \phi-\cos \phi\right), \\
\equiv & R_{\mathrm{DL}+\nabla T} \cos \phi+R_{\mathrm{FL}}+\mathrm{Oe}\left(2 \cos ^{3} \phi-\cos \phi\right) .
\end{aligned}
$$

Here, $R_{\mathrm{PHE}}, R_{\nabla T}$, and $B_{\mathrm{Oe}}$ are the planar Hall resistance, the thermal-induced second-harmonic resistance, and the current-induced Oersted field, respectively. Since both the DL and thermal-induced contributions have the same symmetry, they appear in pairs in Eq. (3); it is also the case for the FL and Oersted field contributions. In this case, as indicated in Eq. (4), we define $R_{\mathrm{DL}+\nabla T}$ and $R_{\mathrm{FL}+\mathrm{Oe}}$ as the coefficients of the $\cos \phi$ and $2 \cos ^{3} \phi-\cos \phi$ components, which can be separated by the fitting of $R_{H}^{2 \omega}$ versus $\phi$. The most convenient way to extract all relevant parameters is to measure both $R_{H}^{1 \omega}$ and $R_{H}^{2 \omega}$ as functions of $\phi$ with various $B_{\text {ext }}$.

Following the above procedure, we extract $B_{\mathrm{DL}}$ and $B_{\mathrm{FL}}$. Representative data of $R_{H}^{1 \omega}$ and $R_{H}^{2 \omega}$, with $B_{\text {ext }}=0.1$ and $0.5 \mathrm{~T}$, in the $\mathrm{IrO}_{2}-T$ sample are exemplified in Figs. 4(a) and 4(b) (top panel), respectively. The amplitude of $R_{H}^{1 \omega}$ 
(a)

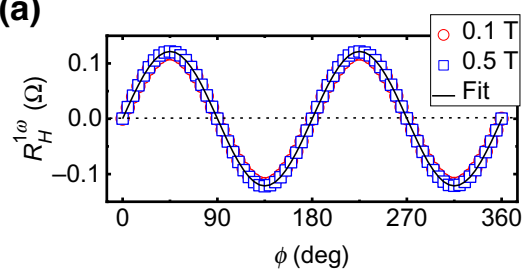

(b)
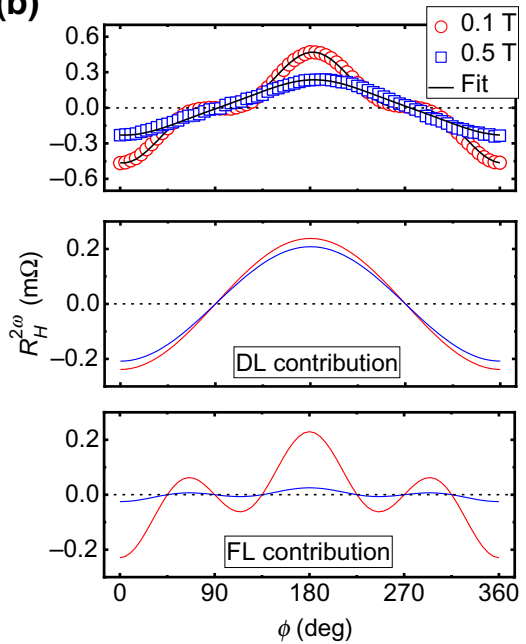

(c)

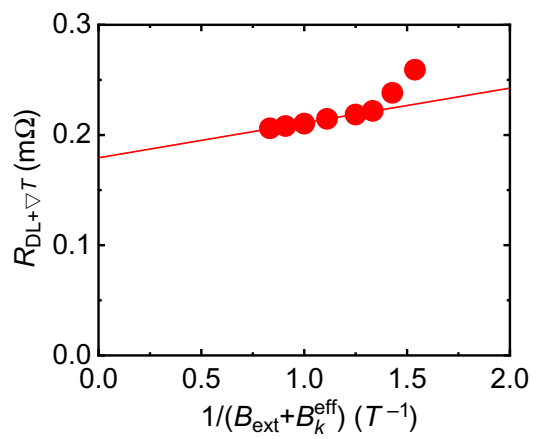

(d)

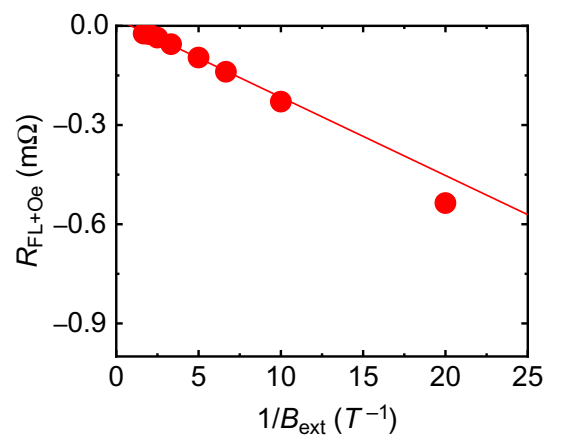

(e)

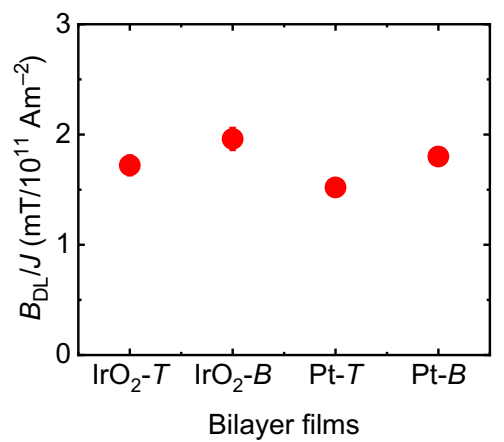

(f)

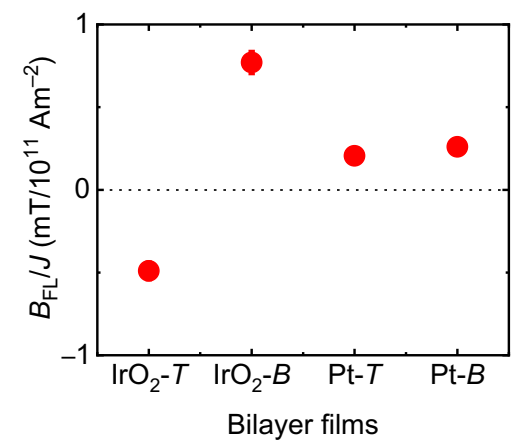

FIG. 4. (a) First-harmonic Hall resistance $\left(R_{H}^{1 \omega}\right)$ of $\mathrm{IrO}_{2}-T$ sample measured at $0.1 \mathrm{~T}$ (red open circle) and $0.5 \mathrm{~T}$ (blue open square). Black solid curve is fitting of data using Eq. (2). (b) Second-harmonic Hall resistance $\left(R_{H}^{2 \omega}\right)$ of $\operatorname{IrO}_{2}-T$ sample measured at 0.1 and $0.5 \mathrm{~T}$ for raw data (top panel). Solid curves are fits to data using Eq. (4). Separated $\cos \phi$ and $2 \cos ^{3} \phi-\cos \phi \operatorname{components~from~} R_{H}^{2 \omega}$ indicate the DL contribution and FL contribution, respectively. (c) Obtained $R_{\mathrm{DL}+\nabla T}$ as a function of $1 /\left(B_{\mathrm{ext}}+B_{k}^{\text {eff }}\right)$ and (d) $R_{\mathrm{FL}}+\mathrm{Oe}$ as a function of $1 / B_{\text {ext }}$ for the $\mathrm{IrO}_{2}-T$ sample. Red solid lines represent linear fits. (e) $B_{\mathrm{DL}} / J$ and (f) $B_{\mathrm{FL}} / J$ for all samples. $J$ is the current density flowing in the nonmagnet layer normalized to $10^{11} \mathrm{~A} / \mathrm{m}^{2}$.

is independent of $B_{\text {ext }}$, indicating that $R_{\mathrm{PHE}}$ is fully saturated magnetization along the in-plane $x-y$ axis at $0.1 \mathrm{~T}$; $R_{\mathrm{PHE}}=0.12 \Omega$ is obtained from the fitting results (black curves) with Eq. (2). On the other hand, $R_{H}^{2 \omega}$ is strongly field dependent, as expected from Eq. (3), reflecting modulation of the SOT. To independently extract $R_{\mathrm{DL}+\nabla T}$ and $R_{\mathrm{FL}+\mathrm{Oe}}$, we fit $R_{H}^{2 \omega}$ by using Eq. (4) to plot components of $\cos \phi$ and $2 \cos ^{3} \phi-\cos \phi$, as DL (middle panel) and FL contributions (bottom panel), respectively. These coefficients are plotted as a function of $1 /\left(B_{\text {ext }}+B_{k}^{\text {eff }}\right)$ and $1 / B_{\text {ext }}$, as shown in Figs. 4(c) and 4(d). The slopes of these curves correspond to $R_{\mathrm{AHE}} B_{\mathrm{DL}}$ and $2 R_{\mathrm{PHE}} B_{\mathrm{FL}+\mathrm{Oe}}$, respectively, from which $B_{\mathrm{DL}}$ and $B_{\mathrm{FL}+\mathrm{Oe}}$ are extracted. To estimate $B_{\mathrm{FL}}$, $B_{\mathrm{Oe}}$ is subtracted from $B_{\mathrm{FL}+\mathrm{Oe}}$ through Ampere's law as $B_{\mathrm{Oe}}=\mu_{0} J t_{\mathrm{NM}} / 2$, where $t_{\mathrm{NM}}$ is the nonmagnet layer thickness and $J$ is the current density flowing in the NM layer given by $J=I /\left(t_{\mathrm{NM}} w\right)$. We exclude two points in the lowfield regime, deviating from the linear relationship, since $M$ is not fully saturated in the $x-y$ plane; $B_{\text {ext }}$ required to saturate the moments is larger than the shape anisotropy field defined by the geometry of the Hall bar [64]. Considering that all samples exhibit similar behavior, the deviation is probably due to the pinning effect, irrespective of oxidation of the Py layer. The intercept of Fig. 4(c) indicates that the thermal effect, $R_{\nabla T}$, is caused by an increase in Joule heating $[11,14,47]$ due to the higher resistance of the $\mathrm{IrO}_{2}$ samples; the Pt samples exhibit a smaller $R_{\nabla T}$ due to their lower resistance. With the parallel resistor model, we determine the current fractions to be $45-50 \%$ for the $\mathrm{IrO}_{2}$ layers and about $88 \%$ for the $\mathrm{Pt}$ layers using each resistivity of $\mathrm{IrO}_{2}, \mathrm{Pt}$, and Py discussed in Sec. III A.

Figures 4(e) and 4(f) display $B_{\mathrm{DL}} / J$ and $B_{\mathrm{FL}} / J$ for all samples, normalized at $J=10^{11} \mathrm{~A} / \mathrm{m}^{2}$, respectively. We find sizable SOT effective fields for all samples, which allow us to evaluate their SOT efficiencies. The sign of DL SOT is positive for all samples, whereas that of FL SOT is positive for the $\mathrm{Pt}$ samples but opposite for the $\mathrm{IrO}_{2}$ samples: negative for $\mathrm{IrO}_{2}-T$ and positive for $\mathrm{IrO}_{2}-B$. The magnitude of the SOT efficiency and the sign change of FL SOT are discussed in next section.

\section{Discussion of spin-orbit-torque efficiency}

The SOT efficiency observed by the two methods (SMR and harmonic Hall) provides an insight to 
(a)

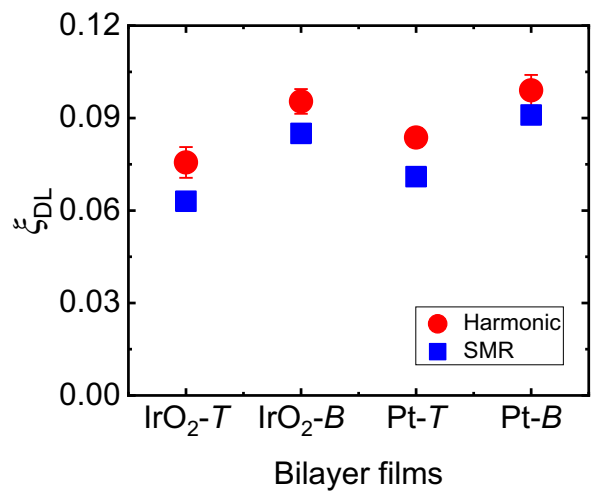

(b)

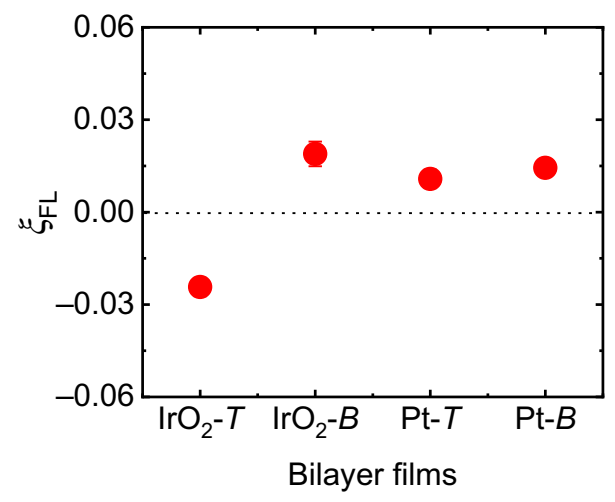

FIG. 5. (a) Dampinglike spinorbit-torque efficiency evaluated by harmonic Hall and spin-Hall magnetoresistance measurements for all samples. (b) Fieldlike spinorbit-torque efficiency for all samples. understand the stacking-order effect of spin-transport properties. The SMR method leads to the DL SOT efficiency $\left(\xi_{\mathrm{DL}}\right)$, while the harmonic Hall method provides both the $\xi_{\text {DL }}$ and FL SOT efficiency $\left(\xi_{\text {FL }}\right)$. Based on the driftdiffusion model, assuming perfect interface transparency, the ratio of SMR can be related to the DL SOT efficiency as follows [24]:

$\Delta R_{z y} / R_{0}=\xi_{\mathrm{DL}}^{2} \frac{\lambda}{t_{\mathrm{NM}}} \frac{\tanh \left(t_{\mathrm{NM}} / 2 \lambda\right)}{1+a}\left[1-\frac{1}{\cosh \left(t_{\mathrm{NM}} / \lambda\right)}\right]$,

where $\lambda$ is spin-diffusion length of the NM layer and $a$ is the current-shunting effect defined as $a \equiv \rho_{\mathrm{NM}} t_{\mathrm{FM}} / \rho_{\mathrm{FM}} t_{\mathrm{NM}}$ due to the presence of the conducting FM layer. $t_{\mathrm{FM}}$ indicates the magnet-layer thickness. Here, we use $\lambda=1.7 \mathrm{~nm}$ for $\mathrm{IrO}_{2}$ [47] and $\lambda=1.4 \mathrm{~nm}$ for Pt [16]. $B_{\mathrm{DL}}$ and $B_{\mathrm{FL}}$ obtained from harmonic Hall measurements can be converted into $\xi_{\mathrm{DL}}$ and $\xi_{\mathrm{FL}}$ according to the following expression [65]:

$$
\xi_{\mathrm{DL}(\mathrm{FL})}=\frac{2 e M_{S} t_{\mathrm{FM}}}{\hbar} \frac{B_{\mathrm{DL}(\mathrm{FL})}}{J},
$$

where $\hbar$ is the Dirac constant and $e$ is the elementary charge. The results of SMR and harmonic Hall methods for all samples are summarized in Figs. 5(a) and 5(b).

First, we discuss $\xi_{\text {DL }}$ determined from both harmonic Hall and SMR methods. In Fig. 5(a), data exhibit similar trends for both methods, while SMR data are smaller than harmonic Hall data; the difference ranges from 8 to $17 \%$. This discrepancy is attributed to other contributions to the observed SMR discussed in Sec. III B. However, qualitative agreement of $\xi_{\mathrm{DL}}$ measured by both methods clearly shows that $\xi_{\text {DL }}$ is mainly from the SMR contribution. We hereafter focus on harmonic Hall data for further discussion. As displayed in Fig. 5(a), harmonic Hall data from 0.076 to 0.100 with positive signs for all samples, which are close to the conventional value of about 0.1 for $\mathrm{IrO}_{2}$ [47] and Pt $[5,6,10,11,14,16,18]$; we consider that the bulk
SHE is the origin of the DL SOT, similar to previous studies $[6,10,11,14-16,18,47]$. This result indicates that the interface of Py and $\mathrm{IrO}_{2}$ plays a significant role in efficient SOT generation.

Second, we find that the stacking order greatly influences $\xi_{\text {DL }}$ in Fig. 5(a). The magnitude of the $\mathrm{IrO}_{2}(\mathrm{Pt})-B$ sample is found to be about 22\% (15\%) larger than that of the $\mathrm{IrO}_{2}(\mathrm{Pt})-T$ one. Previous work on Py-Pt bilayers has shown a similar stacking-order effect on the SOT, which strongly supports our observation [66]. This is attributed to reduced spin transparency in the NM- $T$ samples, since low-spin transparency at the interface typically provides the small SOT generation $[15,16]$. We point out that the situation between the $\mathrm{Pt}$ and $\mathrm{IrO}_{2}$ samples is different in terms of resistance; the Pt samples have the same $R_{\mathrm{sq}}$, while the $\mathrm{IrO}_{2}-T$ sample has a much higher resistance than the $\mathrm{IrO}_{2}-B$ sample due to the oxidized Py layer, as mentioned in Sec. III A. Considering that the variation of $\xi_{\mathrm{DL}}$ is nearly the same in the $\mathrm{IrO}_{2}$ and $\mathrm{Pt}$ samples, the oxidation effect is not the main factor in reducing DL SOT, although it is crucial to increase $R_{\mathrm{sq}}$. A possible factor is the presence of intermixing between FM and NM layers, which could lead to the formation of a nonmagnetic or weakly magnetic surface alloy $[12,22,50]$. In Pt/Co bilayers, by inserting ultrathin spacer layers at the interface, the reduction of DL SOT is more drastic than the increase of $R_{\mathrm{sq}}$, suggesting that the intermixing effect strongly influences the SOT property more than the sample resistance does [22]. Moreover, there are some reports that the deposition of a NM on a FM provides small contributions to interface properties, such as PMA and SOT [19,30,49,50]. Thus, the intermixing effect is likely to be larger in the top NM rather than the bottom NM contacted with the FM as well in our study, although it is hard to separate intermixing from oxidation effects in the $\mathrm{IrO}_{2}$ samples. Following this, the $\mathrm{IrO}_{2}(\mathrm{Pt})-T$ sample exhibits low-spin transparency at the interface, resulting in the reduction of $\xi_{\mathrm{DL}}$. Our experimental results therefore demonstrate the role of the intermixing effect in DL SOT generation in the $\mathrm{IrO}_{2}$ samples by comparing them with the Pt control samples. Here, 
we note that a recent spin-pumping experiment reported an enhancement of the Gilbert damping due to interfacial oxidation in $\mathrm{SrIrO}_{3} / \mathrm{Py}$ bilayers [67], indicating that spincurrent transport might be modified as well. Our observation will be a step to further understand spin-current physics in the $5 d$ transition-metal oxides.

Finally, we discuss the FL SOT in Fig. 5(b). The absolute value of $\xi_{\mathrm{FL}}$ for all samples, ranging from 0.010 to 0.024 , is much smaller than the abovementioned $\xi_{\mathrm{DL}}$, in good agreement with typical bilayers found in Refs. $[11,16,18,47]$. Of particular interest is the opposite sign between the $\mathrm{IrO}_{2}$ samples. Previously, we have reported a negative sign with a possible contribution from the interface REE in $\mathrm{Py} / \mathrm{IrO}_{2}$, which has the same structure as the $\mathrm{IrO}_{2}-T$ sample [47]; similar results are found in metallic bilayers $[16,21,22]$. Our observation in this study emphasizes that the stacking order is crucial for the determination of the sign. This suggests that the opposite sign is not related to the intermixing effect, since the sign for the $\mathrm{Pt}$ samples remains the same. Notably, if the bulk SHE is the dominant source in the FL SOT, the positive signs of both the $\mathrm{IrO}_{2}-B$ and Pt- $B$ samples are reasonable, according to the direction of $\sigma$. Here, we attribute a possible explanation for the negative sign in the $\mathrm{IrO}_{2}-T$ sample to the oxidation effect introduced by inverting the stacking order. The effect causes the formation of $\mathrm{PyO}_{x}$ at the Py surface, enhancing the interfacial REE found in Refs. [68-70]; a theoretical study also suggests that the REE is reinforced by oxidation [71]. If the $\mathrm{PyO}_{x}$ layer, as a thick oxidation barrier, is formed, the spin transparency is significantly decreased, resulting in less SOT generation $[69,72]$. Hence, we find that the oxidation effect plays a major role in FL SOT, in contrast to DL SOT. By inverting the stacking order, our experimental evidence provides a clue for manipulating the sign of FL SOT, with preservation of a large DL SOT, that is, efficient charge-to-spin-current conversion. Finally, we refer to the spin memory loss (SML) at the interface; many efforts so far have been made to quantify the SML in Pt/Py bilayers $[66,73]$, suggesting that it could become another source for changes in the SOT efficiency. Further studies will be required to clarify the microscopic origin of the SOT, such as spin pumping, spin diffusion, and SML.

\section{CONCLUSION}

We investigate the stacking-order effect of spintransport and magnetotransport properties by performing SOT, SMR, and AHE measurements on $\mathrm{Py} / \mathrm{IrO}_{2}$ bilayers. We find a significant stacking-order effect on DL SOT, FL SOT, and PMA. The DL SOT in the $\mathrm{IrO}_{2}(\mathrm{Pt})-B$ sample exhibits a larger magnitude than that in the $\mathrm{IrO}_{2}(\mathrm{Pt})-T$ one, with a positive sign for all samples. The reduction of the $\mathrm{IrO}_{2}(\mathrm{Pt})-T$ sample is attributed to the intermixing effect, reducing spin transparency at the interface; similar behavior in the $\mathrm{IrO}_{2}$ and $\mathrm{Pt}$ samples is observed. The
FL SOT has the opposite sign in the $\mathrm{IrO}_{2}$ samples, in contrast to the same sign in $\mathrm{Pt}$ samples, giving a positive sign for the $\mathrm{IrO}_{2}-B$ and Pt samples and a negative one for the $\mathrm{IrO}_{2}-T$ sample. The negative sign of the FL SOT stems from the interface REE, possibly enhanced by the formation of the $\mathrm{PyO}_{x}$ layer between the $\mathrm{Py}$ and $\mathrm{IrO}_{2}$ layers; the oxidation effect for the $\mathrm{IrO}_{2}-T$ sample plays a major role in FL SOT generation. Finally, we observe that both perpendicular magnetic anisotropy energy density and the anomalous Hall effect are increased in the $\mathrm{IrO}_{2}-B$ sample, suggesting the enhancement of perpendicular anisotropy at the interface; this is similar to results for a typical Pt/FM. Thus, inverting the stacking order significantly influences the interface-driven properties, offering an alternative way to control the sign and magnitude of SOTs as well as magnetic anisotropy in amorphous $\mathrm{IrO}_{2}$-based bilayers. This information is useful for developing the device design, leading to electrically controlled memory and logic devices using $5 d$ transition-metal oxides/FM systems.

\section{ACKNOWLEDGMENT}

The authors thank T. Arakawa for technical support. This work is carried out at the Center for Advanced High Magnetic Field Science at Osaka University under the Visiting Researcher's Program of the Institute for Solid State Physics, University of Tokyo. This work is partly supported by the Japan Society for the Promotion of Science Grant-in-Aid for Young Scientists (Grant No. 19K15434), Scientific Research on Innovative Areas "Quantum Liquid Crystals" (KAKENHI Grant No. JP19H05823), JSTCREST (Grant No. JPMJCR1901), and the Nippon Sheet Glass Foundation for Materials Science and Engineering. We acknowledge stimulating discussions at the meeting of the Cooperative Research Project of the Research Institute of Electrical Communication, Tohoku University.

[1] Y. Otani, M. Shiraishi, A. Oiwa, E. Saitoh, and S. Murakami, Spin conversion on the nanoscale, Nat. Phys. 13, 829 (2017)

[2] A. Manchon, I. M. Miron, T. Jungwirth, J. Sinova, J. Železný, A. Thiaville, K. Garello, and P. Gambardella, Current-induced spin-orbit torques in ferromagnetic and antiferromagnetic systems, Rev. Mod. Phys. 91, 035004 (2019).

[3] A. Hoffmann, Spin Hall effects in metals, IEEE Trans. Magn. 49, 5172 (2013).

[4] V. M. Edelstein, Spin polarization of conduction electrons induced by electric current in two-dimensional asymmetric electron systems, Solid State Commun. 73, 233 (1990).

[5] I. M. Miron, K. Garello, G. Gaudin, P.-J. Zermatten, M. V. Costache, S. Auffret, S. Bandiera, B. Rodmacq, A. Schuhl, and P. Gambardella, Perpendicular switching of a single ferromagnetic layer induced by in-plane current injection, Nature 476, 189 (2011). 
[6] L. Liu, T. Moriyama, D. C. Ralph, and R. A. Buhrman, Spin-Torque Ferromagnetic Resonance Induced by the Spin Hall Effect, Phys. Rev. Lett. 106, 036601 (2011).

[7] L. Liu, C.-F. Pai, Y. Li, H. W. Tseng, D. C. Ralph, and R. A. Buhrman, Spin-torque switching with the giant spin Hall effect of tantalum, Science 336, 555 (2012).

[8] C.-F. Pai, L. Liu, Y. Li, H. W. Tseng, D. C. Ralph, and R. A. Buhrman, Spin transfer torque devices utilizing the giant spin Hall effect of tungsten, Appl. Phys. Lett. 101, 122404 (2012).

[9] J. Kim, J. Sinha, M. Hayashi, M. Yamanouchi, S. Fukami, T. Suzuki, S. Mitani, and H. Ohno, Layer thickness dependence of the current-induced effective field vector in $\mathrm{Ta}|\mathrm{CoFeB}| \mathrm{MgO}$, Nat. Mater. 12, 240 (2013).

[10] K. Garello, I. M. Miron, C. O. Avci, F. Freimuth, Y. Mokrousov, S. Blügel, S. Auffret, O. Boulle, G. Gaudin, and P. Gambardella, Symmetry and magnitude of spin-orbit torques in ferromagnetic heterostructures, Nat. Nanotechnol. 8, 587 (2013).

[11] C. O. Avci, K. Garello, M. Gabureac, A. Ghosh, A. Fuhrer, S. F. Alvarado, and P. Gambardella, Interplay of spin-orbit torque and thermoelectric effects in ferromagnet/normalmetal bilayers, Phys. Rev. B 90, 224427 (2014).

[12] S. Woo, M. Mann, A. J. Tan, L. Caretta, and G. S. D. Beach, Enhanced spin-orbit torques in $\mathrm{Pt} / \mathrm{Co} / \mathrm{Ta}$ heterostructures, Appl. Phys. Lett. 105, 212404 (2014).

[13] T. Nan, S. Emori, C. T. Boone, X. Wang, T. M. Oxholm, J. G. Jones, B. M. Howe, G. J. Brown, and N. X. Sun, Comparison of spin-orbit torques and spin pumping across $\mathrm{NiFe} / \mathrm{Pt}$ and $\mathrm{NiFe} / \mathrm{Cu} / \mathrm{Pt}$ interfaces, Phys. Rev. B 91, 214416 (2015).

[14] C. O. Avci, K. Garello, A. Ghosh, M. Gabureac, S. F. Alvarado, and P. Gambardella, Unidirectional spin Hall magnetoresistance in ferromagnet/normal metal bilayers, Nat. Phys. 11, 570 (2015).

[15] W. Zhang, W. Han, X. Jiang, S.-H. Yang, and S. S. P. Parkin, Role of transparency of platinum-ferromagnet interfaces in determining the intrinsic magnitude of the spin Hall effect, Nat. Phys. 11, 496 (2015).

[16] C. F. Pai, Y. X. Ou, L. H. Vilela-Leao, D. C. Ralph, and R. A. Buhrman, Dependence of the efficiency of spin Hall torque on the transparency of $\mathrm{Pt} /$ ferromagnetic layer interfaces, Phys. Rev. B 92, 064426 (2015).

[17] K. Ueda, M. Mann, C. F. Pai, A. J. Tan, and G. S. D. Beach, Spin-orbit torques in $\mathrm{Ta} / \mathrm{Tb}_{x} \mathrm{Co}_{100-x}$ ferrimagnetic alloy films with bulk perpendicular magnetic anisotropy, Appl. Phys. Lett. 109, 232403 (2016).

[18] Minh-Hai Nguyen, D. C. Ralph, and R. A. Buhrman, Spin Torque Study of the Spin Hall Conductivity and Spin Diffusion Length in Platinum Thin Films with Varying Resistivity, Phys. Rev. Lett. 116, 126601 (2016).

[19] J. Yu, X. Qiu, Y. Wu, J. Yoon, P. Deorani, J. M. Besbas, A. Manchon, and H. Yang, Spin orbit torques and dzyaloshinskii-moriya interaction in dual-interfaced Co-Ni multilayers, Sci. Rep. 6, 32629 (2016).

[20] K. Ueda, M. Mann, P. W. P. de Brouwer, D. Bono, and G. S. D. Beach, Temperature dependence of spin-orbit torques across the magnetic compensation point in a ferrimagnetic TbCo alloy film, Phys. Rev. B 96, 064410 (2017).
[21] Y. Takeuchi, C. Zhang, A. Okada, H. Sato, S. Fukami, and $\mathrm{H}$. Ohno, Spin-orbit torques in high-resistivityW/CoFeB/MgO, Appl. Phys. Lett. 112, 192408 (2018).

[22] C. O. Avci, G. S. D. Beach, and P. Gambardella, Effects of transition metal spacers on spin-orbit torques, spin Hall magnetoresistance, and magnetic anisotropy of Pt/Co bilayers, Phys. Rev. B 100, 235454 (2019).

[23] H. Nakayama, M. Althammer, Y. T. Chen, K. Uchida, Y. Kajiwara, D. Kikuchi, T. Ohtani, S. Geprägs, M. Opel, S. Takahashi, R. Gross, G. E. W. Bauer, S. T. B. Goennenwein, and E. Saitoh, Spin Hall Magnetoresistance Induced by a Nonequilibrium Proximity Effect, Phys. Rev. Lett. 110, 206601 (2013).

[24] Y.-T. Chen, S. Takahashi, H. Nakayama, M. Althammer, S. T. B. Goennenwein, E. Saitoh, and G. E. W. Bauer, Theory of spin Hall magnetoresistance, Phys. Rev. B 87, 144411 (2013).

[25] J. Kim, P. Sheng, S. Takahashi, S. Mitani, and M. Hayashi, Spin Hall Magnetoresistance in Metallic Bilayers, Phys. Rev. Lett. 116, 097201 (2016).

[26] E. Saitoh, M. Ueda, H. Miyajima, and G. Tatara, Conversion of spin current into charge current at room temperature: Inverse spin-Hall effect, Appl. Phys. Lett. 88, 182509 (2006).

[27] K. Ando, S. Takahashi, K. Harii, K. Sasage, J. Ieda, S. Maekawa, and E. Saitoh, Electric Manipulation of Spin Relaxation Using the Spin Hall Effect, Phys. Rev. Lett. 101, 036601 (2008).

[28] P. F. Carcia, Perpendicular magnetic anisotropy in $\mathrm{Pd} / \mathrm{Co}$ and $\mathrm{Pt} / \mathrm{Co}$ thin-film layered structures, J. Appl. Phys. 63, 5066 (1988).

[29] P. Gambardella, S. Rusponi, M. Veronese, S. S. Dhesi, C. Grazioli, A. Dallmeyer, I. Cabria, R. Zeller, P. H. Dederichs, K. Kern, C. Carbone, and H. Brune, Giant magnetic anisotropy of single cobalt atoms and nanoparticles, Science 300, 1130 (2003).

[30] S. Bandiera, R. R. Sousa, B. B. Rodmacq, and B. Dieny, Asymmetric interfacial perpendicular magnetic anisotropy in $\mathrm{Pt} / \mathrm{Co} / \mathrm{Pt}$ trilayers, IEEE Magn. Lett. 2, 3000504 (2011).

[31] Z. B. Guo, W. B. Mi, R. O. Aboljadayel, B. Zhang, Q. Zhang, P. G. Barba, A. Manchon, and X. X. Zhang, Effects of surface and interface scattering on anomalous Hall effect in Co/Pd multilayers, Phys. Rev. B 86, 104433 (2012).

[32] Q. Zhang, P. Li, Y. Wen, C. Zhao, J. W. Zhang, A. Manchon, W. B. Mi, Y. Peng, and X. X. Zhang, Anomalous Hall effect in Fe/Au multilayers, Phys. Rev. B 94, 024428 (2016).

[33] D. Rosenblatt, M. Karpovski, and A. Gerber, Reversal of the extraordinary Hall effect polarity in thin $\mathrm{Co} / \mathrm{Pd}$ multilayers, Appl. Phys. Lett. 96, 022512 (2010).

[34] C. L. Canedy, X. W. Li, and G. Xiao, Large magnetic moment enhancement and extraordinary Hall effect in Co/Pt superlattices, Phys. Rev. B 62, 508 (2000).

[35] G. Y. Guo, Q. Niu, and N. Nagaosa, Anomalous Nernst and Hall effects in magnetized platinum and palladium, Phys. Rev. B 89, 214406 (2014).

[36] N. Nagaosa, J. Sinova, S. Onoda, A. H. MacDonald, and N. P. Ong, Anomalous Hall effect, Rev. Mod. Phys. 82, 1539 (2010). 
[37] R. Wu, C. Li, and A. J. Freeman, Structural, electronic and magnetic properties of $\mathrm{Co} / \mathrm{Pd}(111)$ and $\mathrm{Co} / \mathrm{Pt}(111), \mathrm{J}$. Magn. Magn. Mater. 99, 71 (1991).

[38] W. J. Antel, Jr., M. M. Schwickert, T. Lin, W. L. O’Brien, and G. R. Harp, Induced ferromagnetism and anisotropy of Pt layers in Fe/Pt(001) multilayers, Phys. Rev. B 60, 12933 (1999).

[39] M. Suzuki, H. Muraoka, Y. Inaba, H. Miyagawa, N. Kawamura, T. Shimatsu, H. Maruyama, N. Ishimatsu, Y. Isohama, and Y. Sonobe, Depth profile of spin and orbital magnetic moments in a subnanometer Pt film on Co, Phys. Rev. B 72, 054430 (2005).

[40] K. Fujiwara, Y. Fukuma, J. Matsuno, H. Idzuchi, Y. Niimi, Y. Otani, and H. Takagi, $5 d$ iridium oxide as a material for spin-current detection, Nat. Commun. 4, 2893 (2013).

[41] Z. Qiu, D. Hou, T. Kikkawa, K.-I. Uchida, and E. Saitoh, All-oxide spin seebeck effects, Appl. Phys. Express 8, 083001 (2015).

[42] Y. Sun, Y. Zhang, C.-X. Liu, C. Felser, and B. Yan, Dirac nodal lines and induced spin Hall effect in metallic rutile oxides, Phys. Rev. B 95, 235104 (2017).

[43] A. S. Everhardt, D. C. Mahendra, X. Huang, S. Sayed, T. A. Gosavi, Y. Tang, C.-C. Lin, S. Manipatruni, I. A. Young, S. Datta, J.-P. Wang, and R. Ramesh, Tunable charge to spin conversion in strontium iridate thin films, Phys. Rev. Mater. 3, 051201 (2019).

[44] T. Nan, T. J. Anderson, J. Gibbons, K. Hwang, N. Campbell, H. Zhou, Y. Q. Dong, G. Y. Kim, D. F. Shao, T. R. Paudel, N. Reynolds, X. J. Wang, N. X. Sun, E. Y. Tsymbal, S. Y. Choi, M. S. Rzchowski, Y. B. Kim, D. C. Ralph, and C. B. Eom, Anisotropic spin-orbit torque generation in epitaxial $\mathrm{SrIrO}_{3}$ by symmetry design, Proc. Natl. Acad. Sci. USA 116, 16186 (2019).

[45] H. Wang, K.-Y. Meng, P. Zhang, J. T. Hou, J. Finley, J. Han, F. Yang, and L. Liu, Large spin-orbit torque observed in epitaxial $\mathrm{SrIrO}_{3}$ thin films, Appl. Phys. Lett. 114, 232406 (2019).

[46] P. Jadauna, L. F. Registera, and S. K. Banerjeea, Rational design principles for giant spin Hall effect in $5 d$-transition metal oxides, Proc. Natl. Acad. Sci. USA 117, 11878 (2020).

[47] K. Ueda, N. Moriuchi, K. Fukushima, T. Kida, M. Hagiwara, and J. Matsuno, Spin-orbit torque generation in $\mathrm{NiFe} / \mathrm{IrO}_{2}$ bilayers, Phys. Rev. B 102, 134432 (2020).

[48] A. Bose, J. N. Nelson, X. S. Zhang, P. Jadaun, R. Jain, D. G. Schlom, D. C. Ralph, D. A. Muller, K. M. Shen, and R. A. Buhrman, Effects of anisotropic strain on spin-orbit torque produced by the dirac nodal line semimetal $\mathrm{IrO}_{2}$, ACS Appl. Mater. Interfaces 12, 55411 (2020).

[49] T. Ueno, J. Sinha, N. Inami, Y. Takeichi, S. Mitani, K. Ono, and M. Hayashi, Enhanced orbital magnetic moments in magnetic heterostructures with interface perpendicular magnetic anisotropy, Sci. Rep. 12, 14858 (2015).

[50] I. K. Schuller, S. Kim, and C. Leighton, Magnetic superlattices and multilayers, J. Magn. Magn. Mater. 200, 571 (1999).

[51] M. T. Johnson, P. J. H. Bloemen, F. J. A. den Broeder, and J. J. de Vries, Magnetic anisotropy in metallic multilayers, Rep. Prog. Phys. 59, 1409 (1996).
[52] C. Nistor, T. Balashov, J. J. Kavich, A. Lodi Rizzini, B. Ballesteros, G. Gaudin, S. Auffret, B. Rodmacq, S. S. Dhesi, and P. Gambardella, Orbital moment anisotropy of $\mathrm{Pt} / \mathrm{Co} / \mathrm{AlO}_{\mathrm{x}}$ heterostructures with strong Rashba interaction, Phys. Rev. B 84, 054464 (2011).

[53] T. R. McGuire, J. A. Aboaf, and E. Klokholm, Magnetic and transport properties of Co-Pt thin films, J. Appl. Phys. 55, 1951 (1984).

[54] S. L. Jiang, X. Chen, X. J. Li, K. Yang, J. Y. Zhang, G. Yang, Y. W. Liu, J. H. Lu, D. W. Wang, J. Teng, and G. H. $\mathrm{Yu}$, Anomalous Hall effect engineering via interface modification in Co/Pt multilayers, Appl. Phys. Lett. 107, 112404 (2015).

[55] J. Y. Zhang, Z. L. Wu, S. G. Wang, C. J. Zhao, G. Yang, S. L. Zhang, Y. Liu, S. Liu, J. Teng, and G. H. Yu, Effect of interfacial structures on anomalous Hall behavior in perpendicular $\mathrm{Co} / \mathrm{Pt}$ multilayers, Appl. Phys. Lett. 102, 102404 (2013).

[56] F. Zhang, F. S. Wen, Y. F. Lü, W. Li, Y. F. Lü, Z. Y. Liu, B. Xu, D. L. Yu, J. L. He, and Y. J. Tian, Proper scaling of the anomalous Hall effect in the Co/Pt multilayers, J. Appl. Phys. 110, 033921 (2011).

[57] T. R. Mcguire and R. I. Potter, Anisotropic magnetoresistance in ferromagnetic $3 d$ alloys, IEEE Trans. Magn. 11, 1018 (1975).

[58] A. Philippi-Kobs, A. Farhadi, L. Matheis, D. Lott, A. Chuvilin, and H. P. Oepen, Impact of Symmetry on Anisotropic Magnetoresistance in Textured Ferromagnetic Thin Films, Phys. Rev. Lett. 123, 137201 (2019).

[59] A. Kobs, S. Hesse, W. Kreuzpaintner, G. Winkler, D. Lott, P. Weinberger, A. Schreyer, and H. P. Oepen, Anisotropic Interface Magnetoresistance in $\mathrm{Pt} / \mathrm{Co} / \mathrm{Pt}$ Sandwiches, Phys. Rev. Lett. 106, 217207 (2011).

[60] T. Taniguchi, Magnetoresistance generated from chargespin conversion by anomalous Hall effect in metallic ferromagnetic/nonmagnetic bilayers, Phys. Rev. B 94, 174440 (2016).

[61] M. Kawaguchi, D. Towa, Y. C. Lau, S. Takahashi, and M. Hayashi, Anomalous spin Hall magnetoresistance in Pt/Co bilayers, Appl. Phys. Lett. 112, 202405 (2018).

[62] Y. Yang, Z. Luo, H. Wu, Y. Xu, R. W. Li, S. J. Pennycook, S. Zhang, and Y. Wu, Anomalous Hall magnetoresistance in a ferromagnet, Nat. Commun. 9, 2255 (2018).

[63] W. L. Yang, J. W. Wei, C. H. Wan, Y. W. Xing, Z. R. Yan, X. Wang, C. Fang, C. Y. Guo, G. Q. Yu, and X. F. Han, Determining spin-torque efficiency in ferromagnetic metals via spin-torque ferromagnetic resonance, Phys. Rev. B 101, 064412 (2020).

[64] Y.-C. Lau and M. Hayashi, Spin torque efficiency of Ta, $\mathrm{W}$, and Pt in metallic bilayers evaluated by harmonic Hall and spin Hall magnetoresistance measurements, Jap. Jour. of Appl. Phys. 56, 0802B5 (2017).

[65] A. V. Khvalkovskiy, V. Cros, D. Apalkov, V. Nikitin, M. Krounbi, K. A. Zvezdin, A. Anane, J. Grollier, and A. Fert, Matching domain-wall configuration and spin-orbit torques for efficient domain-wall motion, Phys. Rev. B 87, 020402(R) (2013).

[66] A. J. Berger, E. R. J. Edwards, H. T. Nembach, A. D. Karenowska, M. Weiler, and T. J. Silva, Inductive detection of fieldlike and dampinglike ac inverse spin-orbit torques 
in ferromagnet/normal-metal bilayers, Phys. Rev. B 97, 094407 (2018).

[67] T. S. Suraj, M. Müller, S. Gelder, S. Geprägs, M. Opel, M. Weiler, K. Sethupathi, H. Huebl, R. Gross, M. S. Ramachandra Rao, and M. Althammer, Effect of interfacial oxidation layer in spin pumping experiments on $\mathrm{Ni}_{80} \mathrm{Fe}_{20} / \mathrm{SrIrO}_{3}$ heterostructures, J. Appl. Phys. 128, 083903 (2020).

[68] M. Akyol, J. G. Alzate, G. Yu, P. Upadhyaya, Kin L. Wong, A. Ekicibil, P. K. Amiri, and K. L. Wang, Effect of the oxide layer on current-induced spin-orbit torques in $\mathrm{Hf}|\mathrm{CoFeB}| \mathrm{MgO}$ and $\mathrm{Hf}|\mathrm{CoFeB}| \mathrm{TaO}_{x}$ structures, Appl. Phys. Lett. 106, 032406 (2015).

[69] K. Hasegawa, Y. Hibino, M. Suzuki, T. Koyama, and D. Chiba, Enhancement of spin-orbit torque by inserting $\mathrm{CoOx}$ layer into Co/Pt interface, Phys. Rev. B 98, 020405(R) (2018).
[70] H. Xie, J. Yuan, Z. Luo, Y. Yang, and Y. Wu, In-situ study of oxygen exposure effect on spin-orbit torque in $\mathrm{Pt} / \mathrm{Co}$ bilayers in ultrahigh vacuum, Sci. Rep. 9, 17254 (2019).

[71] P. M. Haney, H.-W. Lee, K.-J. Lee, A. Manchon, and M. D. Stiles, Current induced torques and interfacial spin-orbit coupling: Semiclassical modeling, Phys. Rev. B 87, 174411 (2013).

[72] O. Mosendz, V. Vlaminck, J. E. Pearson, F. Y. Fradin, G. E. W. Bauer, S. D. Bader, and A. Hoffmann, Detection and quantification of inverse spin Hall effect from spin pumping in permalloy/normal metal bilayers, Phys. Rev. B 82, 214403 (2010).

[73] A. J. Berger, E. R. J. Edwards, H. T. Nembach, O. Karis, M. Weiler, and T. J. Silva, Determination of the spin Hall effect and the spin diffusion length of Pt from self-consistent fitting of damping enhancement and inverse spin-orbit torque measurements, Phys. Rev. B 98, 024402 (2018). 